\title{
Author Correction: Linc-DYNC2H1-4 promotes EMT and CSC phenotypes by acting as a sponge of miR-145 in pancreatic cancer cells
}

Yuran Gao', Zhicheng Zhang ${ }^{2}$, Kai Li ${ }^{1}$, Liying Gong ${ }^{1}$, Qingzhu Yang ${ }^{1}$, Xuemei Huang ${ }^{1}$, Chengcheng Hong ${ }^{1}$, Mingfeng Ding ${ }^{2}$ and Huanjie Yang ${ }^{1}$

\section{Correction to: Cell Death \& Disease https://doi.org/10.1038/cddis.2017.311 published online 13 July 2017}

After publication of this article, the below errors were noticed:

The SOX2 primer is incorrect in Table S2.

The Poly( $\mathrm{T})$ adaptor sequence of reverse transcription for miR-145 detection is missing in Table S2.
The corrected table is provided below. This error did not impact the conclusions of the article. We apologize for any confusion or inconvenience to the readers.

Published online: 13 August 2019

Correspondence: Mingfeng Ding (13613604305@163.com) or Huanjie Yang (yanghj@hit.edu.cn)

'School of Life Science and Technology, Harbin Institute of Technology, Harbin, China

${ }^{2}$ Department of General Surgery, Fourth Affiliated Hospital of Harbin Medical University, Harbin, China

These authors contributed equally: Zhicheng Zhang, Kai Li 
Table S2 Primers for qRT-PCR

\begin{tabular}{|c|c|c|}
\hline Targets & \multicolumn{2}{|l|}{ Sequences } \\
\hline Oct4 & F: 5'-CGCCGTATGAGTTCTGTG-3' & R: 5'-GGTGATCCTCTTCTGCTTC-3' \\
\hline $\operatorname{Lin} 28$ & F: 5'-AAAGGAGACAGGTGCTAC-3' & R: 5'-ATATGGCTGATGCTCTGG-3' \\
\hline Nanog & F: 5'-AAGAACTCTCCAACATCCTGAAC-3' & R: 5'-CCTTCTGCGTCACACCATT-3' \\
\hline Sox2 & F: 5'-AGTTGGACAGGGAGATGGC-3' & R: 5'-AACCTTCCTTGCTTCCACG-3' \\
\hline MMP3 & F: 5'-TTCCGCCTGTCTCAAGATGATAT-3' & R: 5'-AAAGGACAAAGCAGGATCACAGTT-3' \\
\hline MMP1 & F: 5'-AAATGCAGGAATTCTITGGG-3' & R: 5'-ATGGTCCACATCTGCTCTTG-3' \\
\hline MMP27 & F: 5'-GTTTAGAAGTGTGGAGCAAAGTCACT-3' & R: 5'-ATAGCGAGGACACCGACCAT-3' \\
\hline ZEB1 & F: 5'-GTTACCAGGGAGGAGCAGTGAAA-3' & R: 5'-GACAGCAGTGTCTTGTTGTTGTAGAAA-3' \\
\hline Vimentin & F: 5'-AGTCCACTGAGTACCGGAGAC-3' & R: 5'-CATTTCACGCATCTGGCGTTC-3' \\
\hline E-cadherin & F: 5'-TACACTGCCCAGGAGCCAGA-3' & R: 5'-TAATCCGGACACTGGTGCCA-3' \\
\hline Linc-DYNC2H1-4 & F: 5'-GCACGGCAGGAAGCAAG-3' & R: 5'-CCAGCCTCCTCTGCCTTGT-3' \\
\hline DYNC2H1-4 & F: 5'-GTGCTGAAAGGGATCGTGTG-3' & R: 5'-GGCTITTGTTGTCATTAGAT-3' \\
\hline GAPDH & F: 5'-TGCACCACCAACTGCTTAGC-3' & R: 5'-GGCATGGACTGTGGTCATGAG-3' \\
\hline U6 & F: 5'-CGCTTCGGCAGCACATATACTA-3' & R: 5'- CGCTTCACGAATTTGCGTGTCA-3' \\
\hline MT-RNR1 & F: 5'-CCTCCCCAATAAAGCTAAAA-3' & R: 5'- GCTATTGTGTGTTCAGATAT-3' \\
\hline \multirow[t]{2}{*}{ miR-145 } & \multicolumn{2}{|c|}{ Poly(T) adaptor: GCTGTCAACGATACGCTACCTAACGGCATGACAGTGTITाTा1ा11111TVN ${ }^{a}$} \\
\hline & F: 5'-GTCCAGTTTTCCCAGGAATCCCT-3' & R: 5'- GCTGTCAACGATACGCTACCTA-3' \\
\hline
\end{tabular}

${ }^{\mathrm{a}} \mathrm{V}=\mathrm{G}, \mathrm{C}, \mathrm{A} ; \mathrm{N}=\mathrm{G}, \mathrm{A}, \mathrm{C}, \mathrm{T}$ 